\title{
Trains of particles in finite-Reynolds-number pipe flow
}

\author{
Jean-Philippe Matas, Virginie Glezer, and Élisabeth Guazzelli \\ IUSTI-CNRS UMR 6595, Polytech' Marseille, Technopôle de Château-Gombert, \\ 13453 Marseille Cedex 13, France \\ Jeffrey F. Morris \\ Halliburton, 3000 N. Sam Houston Parkway East, Houston, Texas 77032
}

(Received 15 April 2004; accepted 22 July 2004; published online 7 October 2004)

In finite-Reynolds-number pipe flow, we have observed long-lived trains of particles aligned with the flow. These trains are located on the Segré-Silberberg equilibrium position which is an annulus close to the wall at the large Reynolds numbers investigated. The effect seems to be controlled by inertia and more precisely by the particle Reynolds number. () 2004 American Institute of Physics.

[DOI: $10.1063 / 1.1791460]$

Inertial effects in uniform streaming flow past bodies are well studied. In sheared suspension flows, this subject is much less understood. While even such basic problems as the flow around individual spherical particles at finite shearflow Reynolds numbers are incompletely understood, it is well known that individual particles undergo inertially driven migration known as the "tubular pinch" effect ${ }^{1}$ in Poiseuille flow. Specifically, at small but finite Reynolds number, experiments showed that a single rigid and neutrally buoyant sphere migrated to an equilibrium position (which we will call the Segré-Silberberg annulus) with its center located at $r=0.6 R$ ( $R$ being the pipe radius) in agreement with asymptotic theory for a channel Poiseuille flow. ${ }^{2}$ When the Reynolds number is increased, the equilibrium position moves toward the wall ${ }^{1,3}$ also in agreement with theoretical predictions. ${ }^{4-6}$ In the course of investigating the Reynolds number dependence of inertial migration, ${ }^{3}$ we discovered a rather curious phenomenon. The spherical particles are found to align in the direction of flow and form long-lived trains of many particles (up to 40). The trains are located on the Segré-Silberberg annulus which has become quite close to the wall at the elevated Reynolds numbers for which they are observed. In fact, "chains" of particles were briefly mentioned by Segré and Silberberg ${ }^{1}$ as a phenomenon occurring when the particle concentration was increased but no systematic study was undertaken.

We studied a neutrally buoyant suspension flowing through a glass tube. We used two sets of polystyrene spheres (Maxi-Blast; South Bend, IN) with mean diameters $d=425 \pm 25 \mu \mathrm{m}$ and $d=825 \pm 25 \mu \mathrm{m}$. The nominal density of the particles is $\rho_{p}=1.05 \mathrm{~g} / \mathrm{cm}^{3}$, for which a suspending liquid $\rho_{f}$ of equal density was formed by mixing glycerol and water, with density matching achieved by finding the mixture in which no sedimentation of a batch of particles occurred. The suspending liquid thus varied slightly around $22 \%$ glycerol and $78 \%$ water at a temperature $T=25^{\circ} \mathrm{C}$, a temperature for which the viscosity of the suspending fluid was in the range $\eta=1.45-1.55 \mathrm{cP}$.

The experimental test section was a horizontal glass tube of length $L=2.6 \mathrm{~m}$. Two tubes of different inner diameters, $D=8 \mathrm{~mm}$ and $D=14 \mathrm{~mm}$, were used. Measurements were undertaken with both sets of particles in the larger tube, giving $D / d=33$ and 17 , and with the smaller particles in the smaller tube, giving $D / d=19$. Defining the pipe-scale Reynolds number as $\operatorname{Re}=\rho_{f} U D / \eta$, with $U$ the mean axial velocity, this length $L=2.6 \mathrm{~m}$ is longer than either of the entry lengths of roughly $50 \mathrm{~cm}$ and $90 \mathrm{~cm}$ needed to obtain fully developed Poiseuille flow at $\operatorname{Re}=2000$ in the $D=8 \mathrm{~mm}$ and $D=14 \mathrm{~mm}$ tubes, respectively. ${ }^{7}$ Above this value of Re, the laminar flow becomes unstable [note that transition at lower $\mathrm{Re}$ is induced by the addition of particles of the scale used here (Ref. 8)]. All results presented are obtained at a measurement position of $1.8 \mathrm{~m}$ from the entrance to the tube. Also, we have not conducted a thorough study of the concentration dependence of the phenomenon, in part because visualization becomes increasingly difficult as the particle fraction increases. The experiments were conducted at $\phi=0.17 \%, 0.06 \%$, and $0.24 \%$ for $D / d=33,17$ and 19 , respectively.

The Poiseuille flow was driven by gravity using continuous overflow from an overhead tank whose elevation was varied. It was not feasible to thermostat the entire system. In order to alleviate settling effects due to density variation with temperature, we mixed the suspension continuously in a thermostated reservoir at the outlet from the test section. From this lower reservoir, the suspension was continuously returned to the overflowing reservoir by a Moineau progressing cavity pump (PCM model MR2.6H24). This arrangement isolated the test section from the pump.

The appearance of the trains of particles was visually obvious above $R e \approx 100$, for the particle and tube diameters used here, with only a few particles in trains at the onset. To illustrate the phenomenon, an image obtained from a condition having relatively long trains is shown in Fig. 1(a). To obtain sufficient information to locate the particles relative to the walls, we have simultaneously imaged from two perspectives in a single frame. This was done by placing a mirror at $45^{\circ}$ relative to the horizontal direction which gives an image of the top of the tube [upper image in Fig. 1(a)]. The lower image of Fig. 1(a) is a view of the side of the tube. We used a digital camera with a frame rate of $25 \mathrm{fps}$. The tube was illuminated by a standard incandescent lamp placed at suffi- 

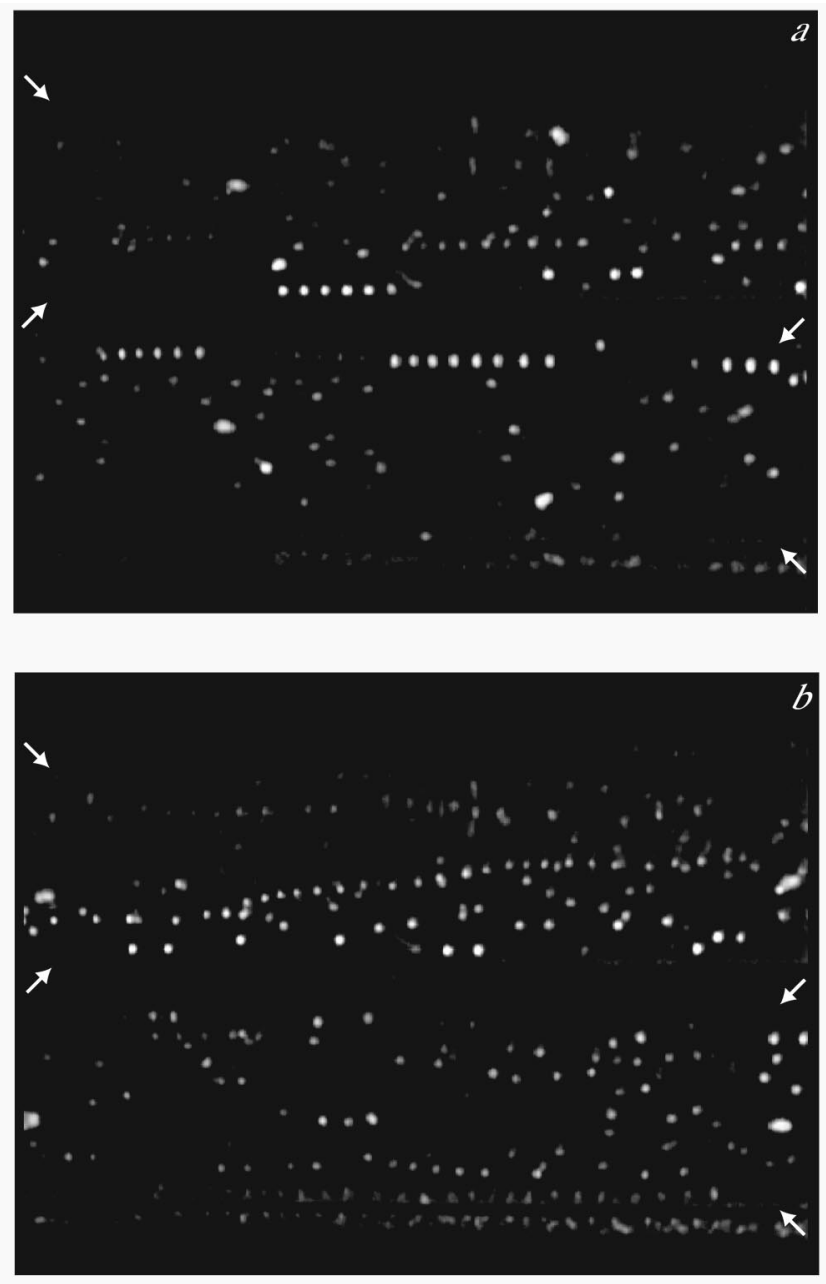

FIG. 1. Photograph showing trains in the pipe from two perspectives for $D / d=19$. (a) $\mathrm{Re}=600$ (in this photograph, the trains are located on the top of the tube and preferentially in the back), (b) $R e=1120$ (in this photograph, one observes a very long train having an angle relative to flow). The arrows indicate the walls of the tube from the two perspectives.

cient distance to avoid heating the tube. The trains are relatively straight on Fig. 1(a), but are also observed at an angle relative to flow, with a typical but long example seen in Fig. 1(b).

Direct counting of the particles identified by eye from the images was used to obtain the data presented in this work. We determined the number of trains observed over a fixed time (typically $30 \mathrm{~s}$ ), as well as the number of particles in each train, defined as a group of three or more aligned particles. We have also measured the surface separations between successive aligned particles. The range of Re studied differed slightly for the various combinations of $d$ and $D$, but was roughly $100 \leqslant \operatorname{Re} \leqslant 2400$. The larger values of Re investigated were above the laminar-intermittent transition, yet short trains of particles were observed in the laminar regions.

The fraction of particles in trains grows with increasing $\mathrm{Re}$, and passes through a maximum depending upon the particle and tube size. This is illustrated in Fig. 2, where the total number of particles in trains normalized by the total number of particles flowing during the measurement time is shown as a function of Re. While the number of particles in

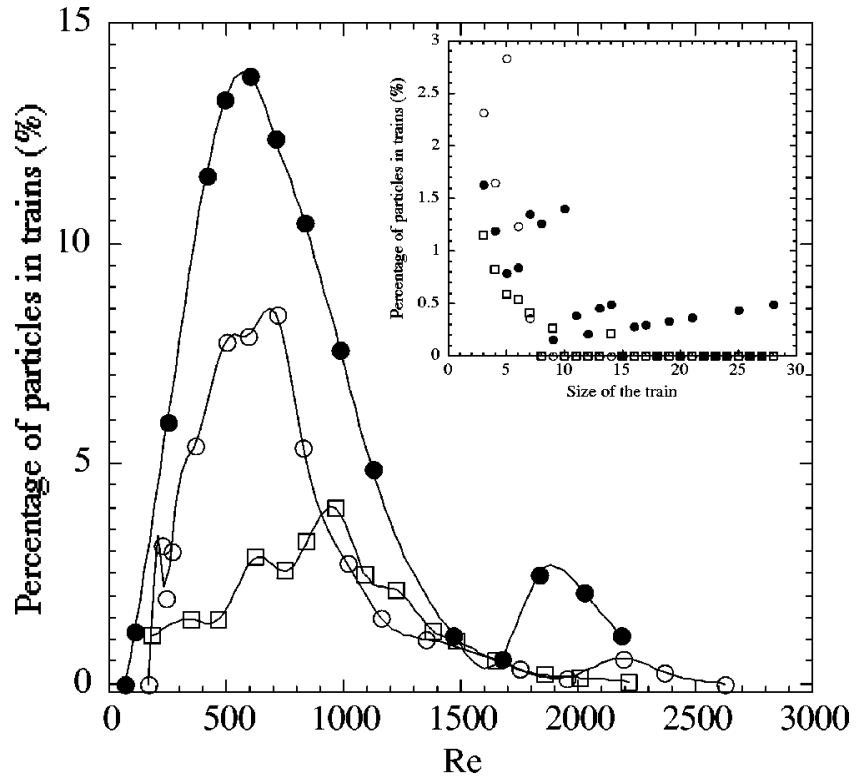

FIG. 2. Percentage of particles in trains as a function of the Reynolds number Re for $D / d=33(\square), 17(\bigcirc)$, and $19(\bullet)$. The lines are used only to guide the eyes.

trains is determined by direct counting, the total number of particles is deduced from the measurement of the particle volume fraction. Because we have studied a constant volume fraction for each $D / d$ and the results are normalized to account for the variation of flowrate, these results indicate that following onset, a progressively larger proportion of particles are in trains as $\mathrm{Re}$ increases, reaches a maximum, and then decreases. The maximum is reached for $D / d=17$ and $D / d$ $=19$ at the same $\operatorname{Re} \sim 700$ and for $D / d=33$ at $\operatorname{Re} \sim 950$. There is also a distribution of particles with respect to length of train in which they are found, as illustrated by the inset to Fig. 2. The inset plot shows the percentage of particles found in trains of a given size, with these measurements made at the Re for which the percentage of particles in all the trains is maximum on the main plot of Fig. 2, for each combination of particle size and tube diameter. There is an overall decrease with the size of the train.

The mean surface separation (normalized by the particle diameter), $l / d$ is found to depend on particle Reynolds number $\operatorname{Re}_{p}=\operatorname{Re}(d / D)^{2}$. To illustrate, we first consider the dependence of $l / d$ as a function of Re, presented in the inset of Fig. 3. Here, the $l / d$ are observed to lie essentially on a single curve for $D / d=17$ and $D / d=19$ particles, decreasing from 3 to 1 particle diameters when Re is varied from 500 to 2000. For $D / d=33, l / d$ remains significantly larger, varying from $l / d \approx 3.5$ down to 2.5 . When the normalized separation is plotted as a function of $\mathrm{Re}_{p}$, as seen in the main plot of Fig. 3, the data collapse quite well to a single curve.

This last finding suggests $\mathrm{Re}_{p}$, determined by the shear flow seen by a particle, controls the interaction between particles within trains. While this quantity could be argued to vary with radial position we have characterized the particlescale inertia under a given condition using the mean shear rate. A possible mechanical basis for the train formation is suggested by the flows around a particle immersed in shear 


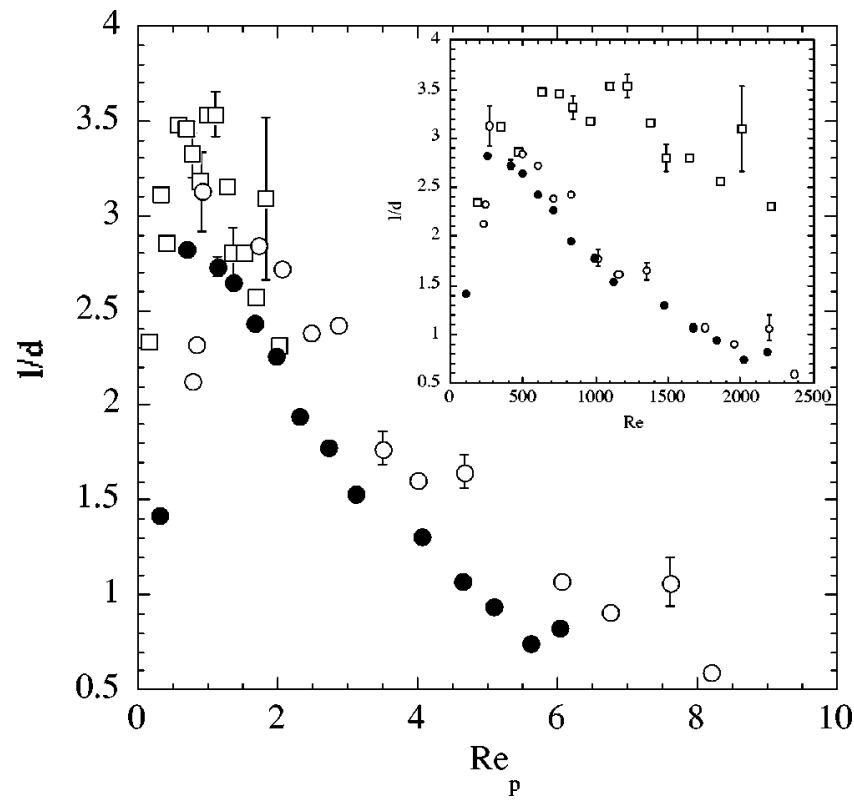

FIG. 3. Mean surface separation normalized by the particle diameter, $l / d$, as a function of $\operatorname{Re}$ (inset) and $\operatorname{Re}_{p}$, for $D / d=33(\square), 17(\bigcirc)$ and $19(\bullet)$. We have indicated statistical error bars for a few data points.

flow. Finite-element flow solutions ${ }^{9}$ have shown that inertia induces (particularly for $\operatorname{Re}_{p} \geqslant 1$ ) a very different flow pattern about a single freely rotating rigid sphere in simple shear. With the caution that the pair-particle flow is not known, we propose that these streamlines may be related to the observed phenomenon. Specifically, when one considers the velocity about the rotating particle, the closed streamline region predicted for $\operatorname{Re}_{p}=0$ collapses rapidly with increase of $\mathrm{Re}_{p}$, and a reverse flow zone appears in which fluid approaches and recedes from the particle in the same direction. Figure 4 shows the analytically determined and numerically predicted streamlines for $\mathrm{Re}_{p}=0$ and $\mathrm{Re}_{p}=10$, respectively. The reverse flow seen at $\operatorname{Re}_{p}=10$, comparable to the largest $\operatorname{Re}_{p}$ we have studied, is similar in form to that observed for smaller finite inertia, and implies passage across the midplane of the particle. Trains are aligned along this midplane, and thus it appears the reverse flow, cut off from recession by the wall, is relevant to trapping in aligned configurations.

The extent of the closed streamline region, and hence also the distance from the reference particle to the boundary of the reversing streamlines, decreases with $\mathrm{Re}_{p}$, a factor which may be related to the decrease in $l / d$ displayed in Fig. 3 . The reverse flow zones extend out of the shear plane (in the vorticity direction), ${ }^{9}$ allowing the possibility for inertial hydrodynamic interactions to result in trains angled relative to the flow. We note that train formation in tube flow is at least visually similar to the well-known concept of drafting in uniform motion at inertial conditions. ${ }^{10}$ In sedimentation at finite Reynolds number, the trailing particle of a pair is observed to catch up with the leading particle. But in contrast to the long-lived trains we have described, it is found in sedimentation that following drafting, there is a tumbling and the kissing pair separates. Note, however, that stable arrangements were seen in thin fluidized beds where three or four spheres could form a permanent structure where each sphere
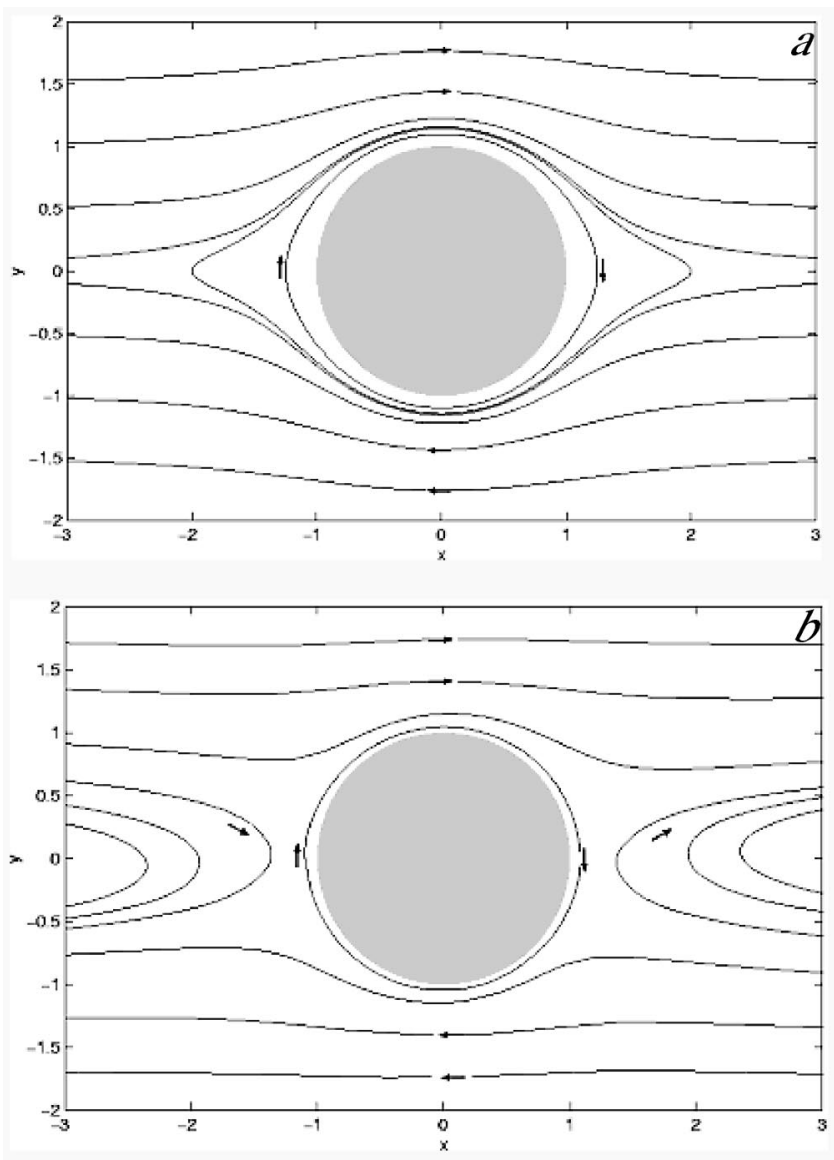

FIG. 4. Streamlines about an isolated sphere (indicated by the solid circle) in unbounded simple shear, $u_{x}=y$ for (a) $\operatorname{Re}_{p}=0$ and (b) $\operatorname{Re}_{p}=10$.

was nested in the wake of the one before and rotated in a shear field there, but only for $22<\operatorname{Re}<43$.

Train formation is influenced, and perhaps constrained, by the radial equilibrium position, the Segré-Silberberg annulus, which moves closer to the wall with increasing Re. Matas et al. $^{3}$ have observed another annulus closer to the center of the pipe which occurs at $\operatorname{Re}>600$. When $\operatorname{Re}$ is increased, the distribution of particles switches from one centered at the Segré-Silberberg annulus to one at this inner annulus, on which no trains are observed. Considering that the suspensions studied are very dilute, this may be due to the fact that this annulus is much broader than the outer Segré-Silberberg annulus and particles are not often forced sufficiently close together. The decrease of the percentage of particles in trains observed after a maximum in Fig. 2 is plausibly related to that effect. On the other hand, the initial increase suggests that the alignment is an inertial effect controlled by $\mathrm{Re}_{p}$.

A small fraction of the larger particles $(d=825 \pm 25 \mu \mathrm{m})$ tends to be flattened by circulations through the pump. Therefore, they may not be perfectly spherical and this may, in principle, affect both the inertial radial migration and the hydrodynamic interactions with other particles. It is plausible that this flattening of a fraction of the particles may explain why the percentage of particles in trains is different in Fig. 2 for the large particles with $D / d=17$ and for the smaller particles with $D / d=19$. In this 
latter case, the percentage of trains is larger and these trains tend to be longer, consistent with the idea that more regular interactions between the more spherical small beads allow more extended trains.

To conclude, we have observed long-lived trains of particles aligned with the flow in the course of investigating the Reynolds number dependence of inertial migration. ${ }^{3}$ The trains are located on the Segré-Silberberg annulus which has become quite close to the wall at the elevated Reynolds numbers for which they are observed. The alignment of particles depends on the particle Reynolds number and is apparently attributable to hydrodynamic interactions associated with the finite-inertia disturbance flows of particles in the shear flow.

The authors thank F. Ratouchniak for technical assistance and Dr. D. R. Mikulencak for Fig. 4. The work was supported in part by the Institut Français du Pétrole, and was undertaken under the auspices of a joint CNRS-NSF research project (CNRS Grant No. 12940; NSF Grant No. 0129079).
${ }^{1}$ G. Segré and A. Silberberg, "Behaviour of macroscopic rigid spheres in Poiseuille flow Part 2. Experimental results and interpretation," J. Fluid Mech. 14, 136 (1962).

${ }^{2}$ B. P. Ho and L. G. Leal, "Inertial migration of rigid spheres in twodimensional unidirectional flows," J. Fluid Mech. 65, 365 (1974).

${ }^{3}$ J.-P. Matas, J. F. Morris, and É. Guazzelli, "Inertial migration of rigid spherical particles in Poiseuille flow," J. Fluid Mech. 515, 171 (2004).

${ }^{4}$ J. A. Schonberg and E. J. Hinch, "Inertial migration of a sphere in Poiseuille flow," J. Fluid Mech. 203, 517 (1989).

${ }^{5}$ A. J. Hogg, "The inertial migration of non-neutrally buoyant spherical particles in two-dimensional shear flows," J. Fluid Mech. 272, 285 (1994).

${ }^{6}$ E. S. Asmolov, "The inertial lift on a spherical particle in a plane Poiseuille flow at large channel Reynolds number," J. Fluid Mech. 381, 63 (1999).

${ }^{7}$ D. J. Tritton, Physical Fluid Dynamics, 2nd ed. (Oxford Science, Oxford, 1988).

${ }^{8}$ J.-P. Matas, J. F. Morris, and É. Guazzelli, "Transition to turbulence in particulate pipe flow," Phys. Rev. Lett. 90, 014501 (2003).

${ }^{9}$ D. R. Mikulencak and J. F. Morris, "Stationary shear flow around fixed and free bodies at finite Reynolds number," J. Fluid Mech. (to be published).

${ }^{10}$ J. Feng, H. Hu, and D. D. Joseph, "Direct simulation of initial value problems for the motion of solid bodies in Newtonian fluid," J. Fluid Mech. 261, 94 (1994). 